\title{
FAUNA DE CHIRONOMIDAE (DIPTERA) ASSOCIADA À Salvinia sp. E Myriophyllum sp. NUM RESERVATÓRIO DO CÓRREGO DO ESPRAIADO, SÃO CARLOS, SÃO PAULO, BRASIL
}

\author{
Carolina Buso Dornfeld \& Alaide A. Fonseca-Gessner
}

\begin{abstract}
Chironomidae (Diptera) fauna associated to Salvinia sp. and Myriophyllum sp. in a Corrego do Espraiado reservoir, São Carlos, São Paulo, Brasil- The presence of vegetation in aquatic ecosystems often results in a substantial increase in the available area for colonization. Several studies have demonstrated a positive relationship between the presence of macrophytes and the abundance and diversity of Chironomidae. Environmental parameters and Chironomidae larvae associated with both macrophytes Salvinia sp. and Myriophyllum sp. were studied in a small reservoir in the state of S. Paulo, Brazil. Tanytarsus, Monopelopia and Labrundinia were dominant in abundance on Salvinia, however Cricotopus and Tanytarsus have alternated dominance on Myriophyllum. In addition the structure functional feeding groups were discussed.
\end{abstract}

Key words: Macrophytes, diversity, abundance

\section{Resumo}

A presença de vegetação num ecossistema aquático é mais uma aréa disponível para a colonização. Vários estudos têm demonstrado

Universidade Federal de São Carlos, Centro de Ciências Biológicas e da Saúde, Departamento de Hidrobiologia, Caixa Postal 676, Rodovia Washington Luís Km 235, CEP: 13565-905 São Carlos, São Paulo, Brasil. E-mail: gessner@power.ufscar.br 
uma relação positiva entre a presença de macrófitas e a abundância e a diversidade de Chironomidae. As variáveis ambientais e as larvas de Chironomidae associadas com as macrófitas Salvinia sp. e Myriophyllum sp. foram estudadas num reservatório com pequenas dimensões no estado de São Paulo, Brasil. Em Salvinia os grupos predominantes foram Tanytarsus, Monopelopia e Labrundinia, entretanto Cricotopus e Tanytarsus alternaram a dominância em Myriophyllum. Alem disso, discutiu-se a estrutura dos grupos funcionais de alimentação associados a essas macrófitas.

Palavras-chave: Macrofitas, diversidade, abundância

\section{Introdução}

A importância das macrófitas aquáticas como um local de permanência de animais aquáticos tem sido reconhecida e amplamente enfatizada por numerosos autores. Todos indistintamente reconhecem a importância da vegetação como um habitat particular de numerosos invertebrados e os estudos da população animal de habitats aquáticos têm usualmente incluído pelo menos alguma referência do animal com a vegetação.

A presença da vegetação num ecossistema aquático resulta num aumento considerável da área disponível para a colonização por invertebrados (Pinder 1995). Beckett et al. (1992), fizeram constatação semelhante quando afirmaram que a função mais importante das macrófitas nos sistemas de água doce para os invertebrados é servir como substrato adicional.

Assim oferecem boas condições de sobrevivência, porque proporcionam abrigo, possibilidades de refúgio contra eventuais predadores, local de oviposição, diversificação de recursos alimentícios, uma vez que as macrófitas também são substrato para algas perifíticas (fonte primária de alimento para muitas espécies fitófagas) e funcionam como filtro retendo partículas orgânicas que podem ser utilizadas pelos organismos detritívoros (Ward, 1992; Trivinho-Strixino \& Strixino, 1993; Trivinho- Strixino et al, 1997).

As larvas de Chironomidae (Diptera) usualmente participam intensamente da zoocenose associada às macrófitas aquáticas, do ponto de vista numérico, como grupo dominante, em diversidade de formas, maior 
número de espécies, e ocupando diferentes níveis tróficos: detritívoros (coletores e filtradores), herbívoros (minadores, raspadores e retalhadores) e predadores (Merritt \& Cummins, 1984; Ward, 1992; Trivinho-Strixino \& Strixino, 1993, 1995; Correia, 1996; Trivinho-Strixino et al,1997).

Este trabalho teve como objetivo estabelecer uma caracterização estrutural e funcional das faunas de Chironomidae que colonizam as macrófitas aquáticas dos gêneros Salvinia sp. e Myriophyllum sp., num reservatório artificial formado pelas águas do Córrego do Espraiado em São Carlos, São Paulo.

\section{Material e Métodos}

O Córrego do Espraiado nasce em uma região de cerrado, próxima ao Campus da Universidade Federal de São Carlos e apresenta boa parte de sua extensão no Parque Ecológico "Dr. Antônio Teixeira Vianna", em São Carlos, SP. Pertence à Bacia Hidrográfica do Rio Monjolinho. Este córrego possui pequenas proporções, e percorre uma área protegida pela vegetação marginal. Parte de sua água é utilizada nos recintos dos animais do Parque Ecológico e parte é utilizada para o abastecimento de água do município de São Carlos.

O reservatório, local de estudo, localiza-se próximo ao ponto de captação de água e dentro do Parque Ecológico, sendo alimentado pelo excedente de água do Córrego do Espraiado que chega ao ponto de captação e pela água proveniente dos recintos dos animais.

As variáveis físicas e químicas foram determinadas, in situ, com um sensor, modelo Horiba-U10. Foram registradas as medidas de: temperatura da água $\left({ }^{\circ} \mathrm{C}\right)$; potencial hidrogeniônico $(\mathrm{pH})$; condutividade $\left(\mu \mathrm{S} . \mathrm{cm}^{-1}\right)$; teor de Oxigênio dissolvido $\left(\mathrm{mg} . \mathrm{l}^{-1}\right)$. A profundidade foi medida utilizando-se um peso amarrado a um corda de náilon com demarcações a cada $0,50 \mathrm{~m}$.

As coletas foram realizadas no período da manhã entre 9:00 e 10:00 horas e a cada coleta obteve-se uma amostra de cada planta com a fauna associada, dentro de um quadrado de $0,50 \mathrm{~m} \times 0,50 \mathrm{~m}$. No laboratório, as amostras de planta foram lavadas sob jato fraco de água, em peneira com malha de $0,20 \mathrm{~mm}$ de abertura e o material retido foi fixado e preservado em álcool a $70 \%$. As larvas dos quironomídeos foram identificadas em gênero e contadas para o cálculo da densidade. 


\section{Resultados}

As medidas de $\mathrm{pH}$, condutividade elétrica, concentração de oxigênio dissolvido, temperatura e profundidade da coluna d'água obtidas junto a cada banco das macrófitas estão apresentadas nas tabelas I e II

Durante o período de estudo, foram realizadas três coletas de cada macrófita (abril, julho e outubro/98) e coletadas 19678 larvas de Chironomidae sendo que dentre as larvas registrou-se a presença de 26 gêneros distribuídos entre cinco tribos (Tabs. III-V).

Para Salvinia sp. três gêneros foram predominantes nos três período de coleta: Tanytarsus, Monopelopia e Labrundinia, e esses gêneros alternaram-se na dominância da fauna de cada mês (Fig. 1).

Para Myriophyllum sp. observa-se que os gêneros dominantes foram Cricotopus (dominante em julho/98) e Tanytarsus (dominante em abril de 1998) (Fig. 2).

Tabela I

Valores das variáveis abióticas registradas junto ao banco de Salvinia

\begin{tabular}{l|ccc}
\hline & \multicolumn{3}{|c}{ Salvinia } \\
Variáveis / Datas & 01.04 .98 & 01.07 .98 & 06.10 .98 \\
\hline $\mathrm{pH}$ & 5,17 & 4,98 & 4,56 \\
Condutividade (us/cm) & 11 & 16 & 35 \\
Oxigênio Dissolvido (mg/l) & 3,13 & 2,08 & 2,73 \\
Temperatura ( C) & 23,4 & 15,63 & 19,3 \\
Profundidade (m) & 0,7 & 0,5 & 0,3 \\
\hline
\end{tabular}

Tabela II

Valores das variáveis abióticas registradas junto ao banco de Myriophyllum

\begin{tabular}{l|ccc}
\hline & \multicolumn{3}{|c}{ Myriophyllum } \\
Variáveis / Datas & 01.04 .98 & 01.07 .98 & 06.10 .98 \\
\hline $\mathrm{pH}$ & 5,65 & 5,41 & 4,51 \\
Condutividade (us/cm) & 11 & 27 & 25 \\
Oxigênio Dissolvido (mg/l) & 3,31 & 5 & 3,31 \\
Temperatura ( C) & 23,6 & 15,8 & 20,9 \\
Profundidade (m) & 1,2 & 0,5 & 0,5 \\
\hline
\end{tabular}


Tabela III

Número de indivíduos pertencentes a cada tribo, associado à Salvinia

\begin{tabular}{l|ccc}
\hline & & Salvinia & \\
Grupos taxonômicos/ Datas & 01.04 .98 & 01.07 .98 & 06.10 .98 \\
\hline Chironomini & 139 & 59 & 20 \\
Tanytarsini & 1118 & 265 & 329 \\
Corynoneurini & 1 & 3 & 2 \\
Orthocladiini & 1 & 2 & 2 \\
Pentaneurini & 1071 & 687 & 571 \\
\hline Total & 2330 & 1016 & 924 \\
\hline
\end{tabular}

Tabela IV

Número de indivíduos pertencentes a cada tribo, associado à Myriophyllum

\begin{tabular}{l|ccc}
\hline & \multicolumn{3}{|c}{ Myriophyllum } \\
Grupos taxonômicos / Datas & 01.04 .98 & 01.07 .98 & 06.10 .98 \\
\hline Chironomini & 450 & 593 & 4892 \\
Tanytarsini & 2405 & 781 & 3765 \\
Corynoneurini & 1 & 49 & \\
Orthocladiini & 405 & 1301 & 186 \\
Pentaneurini & 121 & 95 & 364 \\
\hline Total & 3382 & 2819 & 9207 \\
\hline
\end{tabular}

Quanto aos grupos funcionais, a fauna associada à Salvinia sp. caracterizou-se por apresentar uma predominância de organismos predadores, que contribuíram com $55 \%$ da fauna total coletada, enquanto que em Myriophyllum sp. a categoria predominante foram os coletores, tanto filtradores como roçadores, contribuindo juntos com $84 \%$ da fauna total coletada (Fig. 3).

\section{Discussão}

A água desse reservatório é caracteristicamente ácida, o que reflete o solo de drenagem do tipo cerrado, segundo Maier, (1987), as águas dos rios brasileiros têm $\mathrm{pH}$ com tendência neutra a ácida. A medida de 


\section{Tabela V}

Relação dos gêneros de Chironomidae com as densidades de larvas associadas as macrófitas Salvinia e Myriophyllum.

\begin{tabular}{|c|c|c|c|c|c|}
\hline \multirow[t]{2}{*}{ Chironomidae } & \multicolumn{2}{|c|}{ Salvinia } & \multirow[b]{2}{*}{06.10 .98} & \multicolumn{2}{|c|}{ Myriophylulum } \\
\hline & 01.04 .98 & 01.07 .98 & & 01.04 .98 & 01.07 .98 \\
\hline \multicolumn{6}{|l|}{ Chironominae } \\
\hline \multicolumn{6}{|l|}{ Chironomini } \\
\hline Asheum & 37 & 1 & & 93 & 97 \\
\hline Beardius parcus & 58 & 2 & 1 & 3 & 10 \\
\hline Chironomus gr. decorus & & 16 & & 3 & \\
\hline Chironomus gr. riparius & & & 1 & & \\
\hline Dicrotendipes & 2 & & & 28 & 2 \\
\hline Goeldichironomus sp 1 & & & & 2 & \\
\hline Goeldichironomus pictus & & & & 40 & 18 \\
\hline Goeldichironumus xiborena & & & & & 1 \\
\hline Kiefferullus & 2 & 2 & 4 & 2 & 2 \\
\hline Nilothauma & & & & & 1 \\
\hline Parachironomus & 4 & 31 & 8 & 144 & 179 \\
\hline Parametriocnemus & & 7 & & & \\
\hline Polypedilum & 34 & & 6 & 43 & 280 \\
\hline Stenochironomus & & & & & 1 \\
\hline Tribelos & & & & 1 & 2 \\
\hline Xenochironomus & 2 & & & 91 & \\
\hline \multicolumn{6}{|l|}{ Tanytarsini } \\
\hline Tanytarsini gên. A sp2 & 17 & 89 & 40 & 30 & 113 \\
\hline Tanytarsus & 1101 & 176 & 289 & 2375 & 668 \\
\hline \multicolumn{6}{|l|}{ Orthocladiinae } \\
\hline Corynoneura & 1 & 3 & 2 & 1 & 49 \\
\hline Cricotopus & 1 & 2 & 2 & 405 & 1301 \\
\hline \multicolumn{6}{|l|}{ Tanypodinae } \\
\hline Ablabesmyia & 1 & 13 & 4 & 21 & 14 \\
\hline Gr. Thienemannimyia & 6 & & & & \\
\hline Labrundinia & 296 & 337 & 431 & 70 & 81 \\
\hline Larsia & & & 13 & 9 & \\
\hline Monopelopia & 768 & 337 & 113 & 21 & \\
\hline Pentaneura & & & 10 & & \\
\hline Total & 2330 & 1016 & 924 & 3382 & 2819 \\
\hline
\end{tabular}



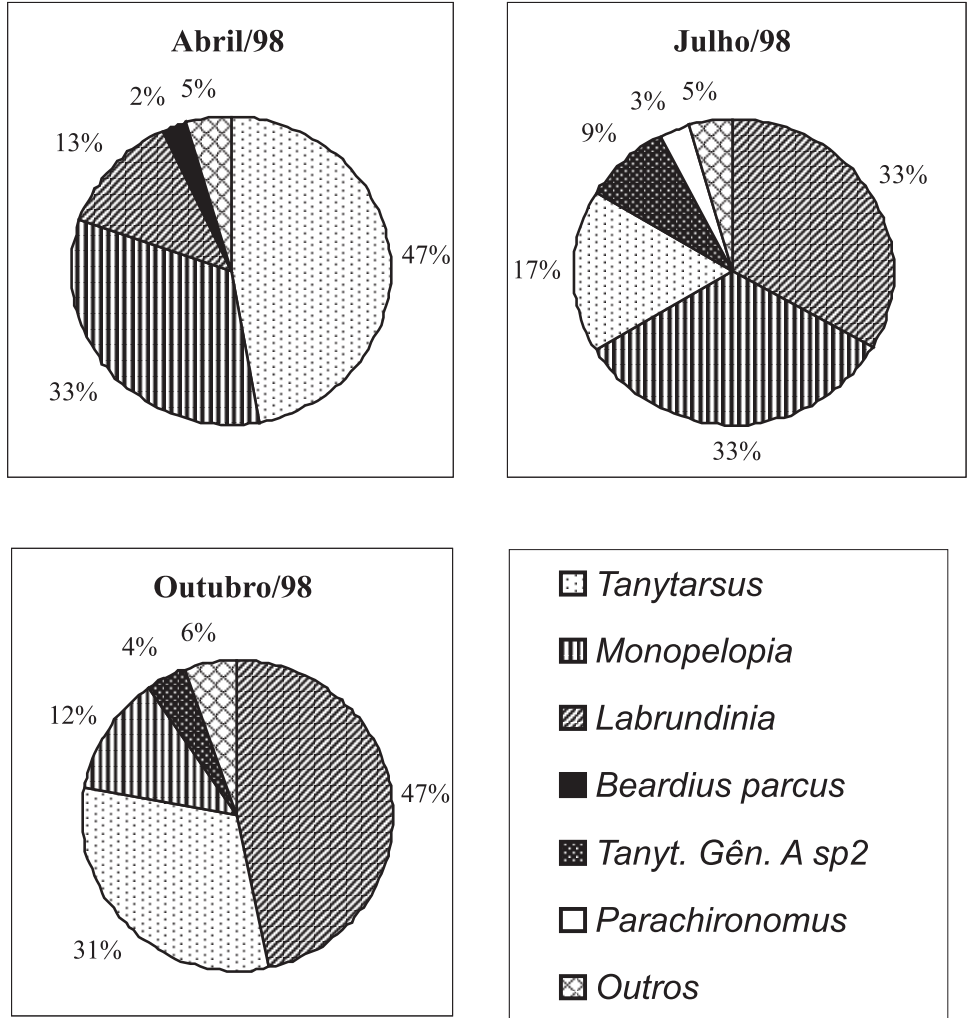

⿴囗十 Tanytarsus
m Monopelopia
m Labrundinia
Beardius parcus
Tanyt. Gên. A sp2
$\square$ Parachironomus
⿴囗utros

Figura 1: Gêneros de Chironomidae associados à Salvinia.
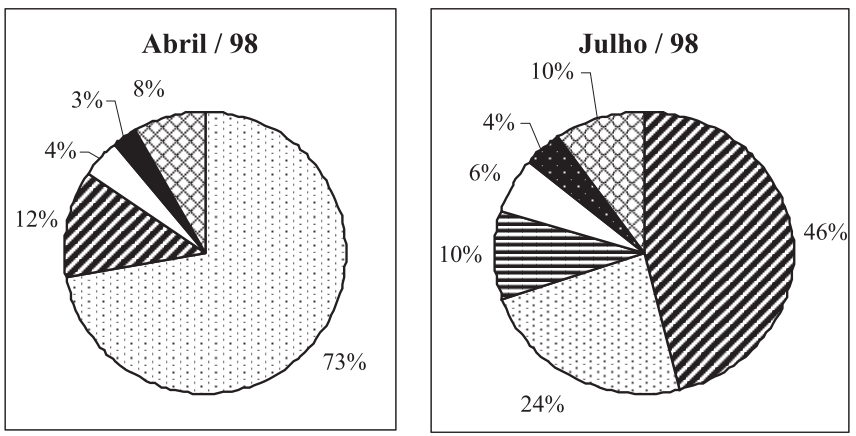

日 Tanytarsus
UCricotopus
$\square$ Parachironomus sp
$\square$ Xenochironomus
目Polypedilum
- Tanyt. Gên. A sp2
¿ Outros

Figura 2: Gêneros de Chironomidae associados à Myriophyllum. 

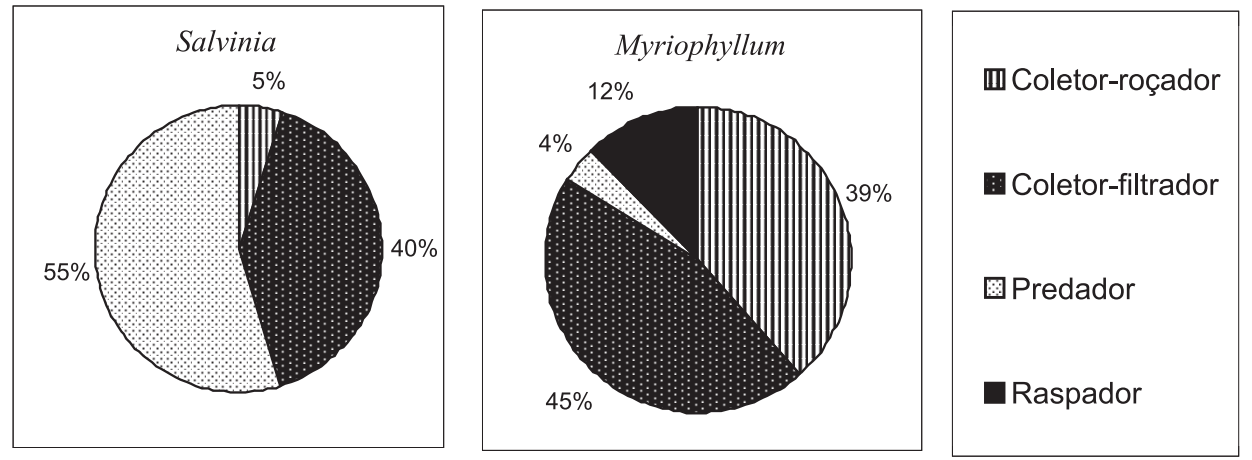

Figura 3: Categorias funcionais de alimentação das larvas de Chironomidae associadas às macrófitas Salvinia e Myriophyllum.

condutividade elétrica revela a quantidade total de matéria ionizada (Maier, 1987), bem como fornece informações sobre o metabolismo do ecossistema aquático (Esteves, 1988). No reservatório a condutividade elétrica é baixa a muito baixa, refletindo que o sistema não sofre interferência antrópica significativa. As concentrações de oxigênio dissolvido apresentaram-se menores para a água junto ao banco de Salvinia e este fato pode ser devido à formação de densos bancos dessa macrófita que impedem a passagem de luz e consequentemente diminui o crescimento e desenvolvimento de microalgas reduzindo, dessa forma, a fotossíntese. Além disso, as folhas fotossintetizantes de Salvinia ficam acima do nível da água e portanto o oxigênio produzido vai para a atmosfera, enquanto que Myriophyllum possui folhas fotossintetizantes submersas que contribui para o aumento do oxigênio dissolvido na água. A temperatura da água segue um padrão típico de variação sazonal para a região, tendo sido registrado o valor mínimo de 15,6 ํ em julho/98 e o valor máximo de 23,6 ${ }^{\circ} \mathrm{C}$ em abril/98. A temperatura tem influencia direta sobre a dissolução de oxigênio na água e na comunidade biótica, pois muitos parâmetros biológicos, especialmente crescimento larval, tamanho do adulto, fecundidade e tempo de eclosão dos ovos são significantemente afetados pela temperatura. A temperatura mais baixa foi registrada no mês de julho no qual houve uma menor quantidade de organismos associados às macrófitas. A profundidade máxima registrada no mês de abril, coincide com o período de chuvas dessa região, o que faz com que a profundidade da coluna d'água aumente. Porém mesmo nesse período chuvoso o 
reservatório pode ser considerado raso por possibilitar o desenvolvimento de plantas submersas como o Myriophyllum.

A predominância de Chironomidae vivendo associados à macrófitas aquáticas tem sido salientada em diversos trabalhos realizados em ambiente lênticos (Strixino \& Trivinho-Strixino, 1984; Pinder, 1986; Correia, 1996; Trivinho-Strixino et al., 1997).

Uma das características que propiciam o estabelecimento da fauna de Chironomidae é a omnivoria e a variabilidade de obtenção de alimentos, tendo representantes em diversas categorias tróficas (Có, 1994 apud Correia, 1996).

Ao analisarmos os gêneros de Chironomidae associados à Salvinia sp. pode-se observar que três gêneros predominam nas coletas : Tanytarsus, Monopelopia e Labrundinia. Os dois últimos são predominantemente predadores e a associação de organismos predadores associados à Salvinia sp. foi considerada anteriormente. Segundo Wetzel, 1975, entre as larvas de Chironomidae predadoras, o jovem no primeiro instar são detritívoras, mas a partir do segundo ínstar elas substituem seu alimento por pequenas larvas de outros Chironomidae, Oligochaeta e pequenos crustáceos.

Quando se observa os gêneros de Chironomidae associados à Myriophyllum sp., verifica-se que Tanytarsus e Cricotopus são os gêneros com maior densidade.

A presença de Cricotopus associada a essa macrófita já foi mencionada por alguns autores e segundo Kangasniemi \& Oliver (1983), esse gênero causaria danos a macrófita por se alimentar dos bulbos apicais submersos, exercendo, dessa maneira, o papel de agente controlador biológico do crescimento dessa macrófita. BERG, 1995, considera esse gênero com raspador e não retalhador como consideram Kangasniemi \& Oliver, 1983 e Merritt \& Cummins, 1984. Corroborando com a hipótese de BERG, 1995, DIAS em 1998, constatou em seu estudo somente a presença de detritos e algas no conteúdo estomacal das larvas de Cricotopus. Strixino \& Trivinho-Strixino, 1984, acreditam que as considerações de Kangasniemi \& Oliver, 1983, deveriam merecer estudos mais apronfudados.

Outro gênero associado à Myriophyllum sp. que merece ser considerado é Xenochironomus. Segundo Trivinho-Strixino \& Strixino, 1995, as larvas desse gênero são minadoras de esponjas de água doce e em concordância com este fato, registrou-se a presença de esponjas associadas ao Myriophyllum sp. somente no mês de abril/98, quando este gênero foi coletado. 
Tanytarsus esteve presente em grande quantidade em todas as coletas nas duas macrófitas. A presença desse táxon possivelmente está relacionada com o grande aporte de matéria orgânica que chega ao reservatório, vinda essencialmente dos recintos dos animais do parque ecológico. As "raízes" de Salvinia, assim como os folíolos submersos de Myriophyllum sp. podem funcionar como filtros retendo matéria orgânica, favorecendo o crescimento e desenvolvimento de organismos coletores.

Levando-se em consideração que $84 \%$ dos organismos associados ao Myriophyllum sp. são coletores (filtradores e roçadores) pode-se supor que em termos de retenção de matéria orgânica, os folíolos de Myriophyllum sp. são mais eficientes do que as "raízes" de Salvinia sp.

Analisando as categorias funcionais, encontra-se diferenças entre a composição da fauna associada as duas macrófitas. Enquanto os predadores foram dominantes em Salvinia sp. com 55\%, em Myriophyllum sp. a participação de organismos desta categoria não ultrapassou $4 \%$ da fauna total. Trivinho-Strixino et al. (1997), também constataram elevada participação de predadores em Salvinia sp. em lagoas da Estação Ecológica de Jataí, Luiz Antônio, São Paulo.

Em Myriophyllum sp. as categorias predominantes foram coletorfiltrador e coletor-roçador A grande porcentagem de Chironomidae coletores, um grupo detritívoro, constiui forte evidência do predomínio da cadeia de detritos, um fenômeno geralmente resultante do "enriquecimento trófico" (Mastrantuono, 1986).

Segundo Mc Lachlan (1969) essas diferenças entre a fauna associada as duas macrófitas, podem estar correlacionadas com a densidade dos bancos de macrófitas e, portanto com a penetração de luz. O mesmo autor, em estudo similar, observou que uma grande população de Chironomidae estava presente no vegetal submerso (Potamogeton pusillis), onde a penetração de luz era boa, enquanto que uma menor população esteve presente sob o denso banco do vegetal flutuante (Salvinia auriculata). Verificou-se também que grande parte dos Chironomidae era da subfamília Tanypodinae, que parece conter membros predadores e predadores facultativos. A predominância de predadores associados à Salvinia sp. sugere a utilização do vegetal como um esconderijo (Karassoska, 1960 apud Mc Lachlan, 1969), onde os animais podem exercer melhor suas estratégias de caça. A dificuldade de penetração de luz, proporcionada pelo denso banco de Salvinia sp., pode resultar na ausência de microalgas e portanto, o único recurso alimentar para os 
organismos, seria os detritos retidos nas "raízes" de Salvinia sp. Mas devido a sua rizosfera reduzida o acúmulo de detrito é dificultado impedindo, assim, a presença de alguns grupos (Trivinho-Strixino et al., 1997).

\section{Referências Bibliográficas}

Beckett, D.C., Aatila, T.P. \& Miller, A.C., 1992 Invertebrate abundance on Potamogeton nodosus, effects of plant surface area na condiction. Can. J. Zool. 70:300-306

Berg, M.B., 1995, Larval food na feeding behaviour p. 136-168. In: The Chironomidae, the biology and ecology of non-biting midges. Armitage, P.P.S.. Granston \& Pinder. Ed. Chapman \& Hall. 572 p

Correia, L., 1996, Estrutura e Função da Fitofauna Associada à Rizosfera de Scirpus cubensis na Lagoa do Infernão (Reserva Ecológica do Jataí - SP). Monografia apresentada ao CCBS - UFSCar, São Carlos, São Paulo. 43 p.

Correll, D.S. \& Correll, H.B., 1975, Aquatic and Wetland Plants of Southwestern United States. Stanford University Pres, Stanford, California, II. 1777 p.

Dias, A.C., 1998 Estudo da Fauna de Insetos Aquáticos Associados a Diferentes Substratos no Córrego do Beija-Flor na Estação Ecológica do Jataí, Luiz Antônio, SP - Relações Tróficas das Larvas de Chironomidae (Diptera). Monografia apresentada ao CCBS UFSCar, São Carlos, São Paulo. 48 p

Esteves, F.A., 1988, Fundamentos de Limnologia. Interciência/FINEP, Rio de Janeiro, $575 \mathrm{p}$.

Kangasniemi, B.J. \& Oliver, D.R., 1983, Chironomidae (Diptera) Associated with Myriophyllum spicatum in Okanagan Valley Lakes, British, Columbia. Can. Entomol. 15:1545-1546. 
Maier, M.H., 1987. Ecologia da bacia do rio Jacaré-Pepira (47ํ5' $-48^{\circ} 55^{\prime} \mathrm{W}$;

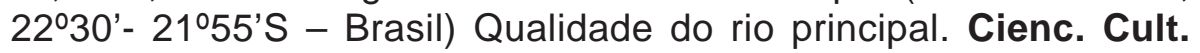
39:164-185.

Mastrantuono, L., 1986. Community Struture of the Zoobenthos Associated With Submerged Macrophytes in the Eutrophic Lake Nemi (Central Italy). Bool. Zool. 53:41-47.

Mc Lachlan, A.J., 1969. The Effect of Aquatic Macrophytes on the Variety and Abundance of Benthic Fauna in a Newly Created Lake in the Tropics (Lake Kariba). Arch. Hydrobiol. 62:212-231.

Merritt, R.W. \& Cummins, K.W. (eds.), 1984. An Introdution to the Aquatic Insects of the North America. Kendall/Hunt Publishing Co., Dubuque, lowa. $772 \mathrm{p}$.

Pinder, L.C.V., 1986, Biology of Freshwater Chironomidae. Ann. Rev. Entomol. 31:1-23.

Santos, J.E., Paese, A. \& Pires, J.S.R., 1998, Unidades da Paisagem (Biótopos) do campus da UFSCar, PPG- ERN, UFSCar, São Carlos, São Paulo. 11 p

Strixino, G.B.M.A. \& Strixino, S.T., 1984, Macroinvertebrados Associados a Tapetes Flutuantes de Eichhornia crassipes (Mart.) Solms, de um Reservatório. Ann. Sem. Reg. Ecol. 4:375-397.

Trivinho-Strixino, S. \& Strixino, G.B.M.A., 1995, Larvas de Chironomidae (Diptera) do Estado de São Paulo: Guia de Identificação e Diagnose dos Gêneros, PPG-ERN/ UFSCar. São Carlos, São Paulo. 229 p

Trivinho-Stixino, S; Gessner, A.F. \& Correia, L., 1997. Macroinvertebrados Associados a Macrófitas Aquáticas as Lagoas Marginais da Estação Ecológica do Jataí (Luiz Antônio - SP). Anais do VIII Sem. Reg.Ecol. 8:53-60

Ward, J.V., 1992. Aquatic Insect Ecology, Jonh Wiley \& Sons, Inc. 424 p.

Wetzel, R.G., 1975. Limnology. Saunders College Publishing Philadelphia. $743 p$. 